\title{
Avaliação clínica da maturação da fístula arteriovenosa para hemodiálise: revisão de escopo
}

Arteriovenous fistula maturation clinical assessment for hemodialysis: a scoping review Evaluación clínica de la maduración de la fístula arteriovenosa para hemodiálisis: revisión de alcance

Bianca Rafaela Correia ${ }^{1}$ io https://orcid.org/0000-0001-7517-4820

Marcos Antonio Gomes Brandão ${ }^{1}$ io https://orcid.org/0000-0002-8368-8343

Rafael Oliveira Pitta Lopes ${ }^{1}$ id https://orcid.org/0000-0002-9178-8280

Paulo Cezar Gonçalves da Silva ${ }^{1}$ io https://orcid.org/0000-0001-8711-8164

Kênia Rocha Leite Zaccaro io https://orcid.org/0000-0002-0251-1651

Andrea Barroso Benevides ${ }^{1}$ io https://orcid.org/0000-0002-9035-5766

Sabrina da Costa Machado Duarte ${ }^{1}$ io https://orcid.org/0000-0001-5967-6337

Rafael Celestino da Silva ${ }^{1}$ io https://orcid.org/0000-0002-5211-9586

Correia BR, Brandão MA, citar:

Zaccaro KR, Benevides AB, et al. Avaliação clínica da maturação da fístula arteriovenosa para hemodiálise: revisão de escopo. Acta Paul Enferm. 2021;34:eAPE00232.

DOI

http://dx.doi.org/10.37689/actaape/2021AR00232

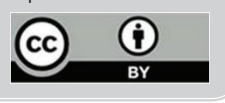

Fístula arteriovenosa; Diálise renal; Avaliação em saúde; Avaliação em enfermagem; Cuidados

pós-operatórios

Keywords

Arteriovenous fistula; Renal dialysis; Health evaluation; Postoperative care

Descriptores

Fístula arteriovenosa; Diálisis renal; Evaluación en salud; Cuidados posoperatorios

Submetido 8 de Fevereiro de 2020

Aceito

17 de Junho de 2020

Autor correspondente

Bianca Rafaela Correia

E-mail: bianca.rafaela.c@gmail.com

\section{Resumo}

Objetivo: Mapear as evidências de pesquisa disponíveis para avaliação clínica da maturação da fístula arteriovenosa.

Métodos: Estudo do tipo Scoping review, com busca realizada entre outubro a novembro de 2019 nas bases de dados JBI, Cochrane, Biblioteca Virtual em Saúde, PubMed, e CINAHL, sendo incluídos estudos com pacientes maiores de 18 anos, com doença renal crônica pré-dialítica ou já em hemodiálise, submetidos à cirurgia da fístula arteriovenosa; texto disponível; e idiomas inglês, espanhol ou português. Foram excluídos estudos relacionados a fase pós-operatória ou relacionados a cuidados com próteses/enxertos arteriovenosos.

Resultados: Foram identificadas 1954 publicações elegíveis, dos quais 38 compuseram a amostra final. 0 estudo compreendeu publicações entre 1998 e 2018, com abrangência internacional do tema (94,7\%). 0 profissional executor da avaliação foi predominantemente 0 enfermeiro ou equipe de enfermagem $(47,4 \%)$, e a avaliação teve início no pós-cirúrgico imediato, até meses após a cirurgia. Dentre as técnicas de avaliação clínica, 23 estudos (60,5\%) recomendaram o exame físico e 15 (39,5\%) a combinação anamnese e exame físico.

Conclusão: A presente revisão apresentou o mapeamento de evidências, nas quais a avaliação clínica da fístula arteriovenosa deve incluir anamnese e exame físico, sendo apresentados vários elementos ligados a permeabilidade e respostas vasculares do acesso. Houve a predominância do profissional enfermeiro como avaliador, sendo ressaltada a necessidade do treinamento para execução da avaliação, além de uma avaliação mais abrangente, com aplicação do Processo e Teorias de Enfermagem, e Linguagens Padronizadas, o que pode propiciar um novo campo de investigação e desenvolvimento na área.

\section{Abstract}

Objective: To map the available research evidence for arteriovenous fistula maturation clinical assessment.

Methods: A scoping review, with a search conducted between October and November 2019 at JBI, Cochrane, Virtual Health Library, PubMed, and CINAHL. Studies with patients over 18 years old, with pre-dialysis chronic kidney disease or already under hemodialysis, who underwent arteriovenous fistula surgery were included; available text; and in English, Spanish, and Brazilian Portuguese. Studies related to the postoperative phase or related to care with prostheses/arteriovenous grafts were excluded.

Results: One thousand nine hundred and fifty-four eligible studies were identified; 38 made up the final sample. The study comprised studies between 1998 and 2018, with an international scope of the theme $(94.7 \%)$. The professional who performed the assessment was predominantly the nurse or nursing team $(47.4 \%)$, and assessment started in the immediate post-surgical period, even months after surgery. Among 
the clinical assessment techniques, 23 studies (60.5\%) recommended physical examination and $15(39.5 \%)$ the combination of medical history and physical examination.

Conclusion: This review presented the mapping of evidence, in which arteriovenous fistula clinical assessment must include medical history and physical examination. Several elements related to the permeability and vascular responses of the access were presented. There was a predominance of professional nurses as evaluators. There was need for training to carry out the assessment, in addition to a more comprehensive assessment, with the application of the Nursing Theory and Standardized Languages and Process, which may provide with a new field of research and development in the area.

\section{Resumen}

Objetivo: Mapear las evidencias de investigación disponibles para evaluación clínica de la maduración de la fístula arteriovenosa.

Métodos: Estudio tipo scoping review, cuya búsqueda fue realizada entre octubre y noviembre de 2019 en las bases de datos JBI, Cochrane, Biblioteca Virtual em Saúde, PubMed y CINAHL. Se incluyeron estudios con pacientes mayores de 18 años, con enfermedad renal crónica predialítica o ya en hemodiálisis, sometidos a cirugía de fístula arteriovenosa y textos disponibles en inglés, español o portugués. Se excluyeron estudios relacionados con la fase posoperatoria 0 relacionados con cuidados de prótesis/injertos.

Resultados: Se identificaron 1.954 publicaciones elegibles, de las cuales 38 formaron parte de la muestra final. El estudio comprendió publicaciones entre 1998 y 2018, con alcance internacional del tema (94,7 \%). El profesional que ejecutó la evaluación fue predominantemente el enfermero 0 el equipo de enfermería $(47,4 \%$ y la evaluación comenzó en el posoperatorio inmediato, hasta meses después de la cirugía. Entre las técnicas de evaluación clínica, 23 estudios (60,5\%) recomendaron el examen físico y 15 (39,5 \%) la combinación entre anamnesis y examen físico.

Conclusión: La revisión presentó el mapeo de evidencias, en las cuales la evaluación clínica de la fístula arteriovenosa debe incluir anamnesis y examen físico. También se presentaron varios elementos relacionados con la permeabilidad y respuestas vasculares del acceso. Hubo una predominancia del profesional enfermero como evaluador y se destaca la necesidad de entrenamiento para llevar a cabo la evaluación, además de una evaluación más abarcadora, con aplicación del proceso y teorías de enfermería y un lenguaje estandarizado, lo que puede proporcionar un nuevo campo de investigación y desarrollo del área.

\section{Introdução}

Dentre as modalidades de terapia de reposição renal para lidar com doença renal crônica (DRC), a hemodiálise é a principal, alcançando $70 \%$ a $90 \%$ dos pacientes, com todos necessitando de adequado acesso vascular. ${ }^{(1,2)}$ As alternativas são a Fístula Arteriovenosa (FAV) e os Cateteres Venosos Centrais (CVC), sendo a FAV considerada o padrão-ouro por melhores indicadores para risco de infecçóes, hospitalizaçôes, ocorrência de estenose venosa central, mortalidade e custos do que o CVC. ${ }^{(3-7)}$ Então, as estratégias de criação e acompanhamento da Fístula Arteriovenosa tornam-se relevantes. ${ }^{(2,8,9)}$

A FAV é uma anastomose autógena entre uma artéria e uma veia. Após sua criação, um fluxo contínuo da artéria para veia inicia uma série de mudanças, alterando a estrutura da parede, gerando uma tensão de cisalhamento, e aumentando rapidamente o fluxo sanguíneo durante as primeiras 24 horas. Essas mudanças tornam a FAV adequada para suportar repetidas punçôes da terapia dialítica; devendo passar pela fase de maturação, que leva cerca de quatro a seis semanas, envolvendo mudanças como aumento do fluxo sanguíneo para $500 \mathrm{ml} / \mathrm{min}$, diâmetro mínimo de $4 \mathrm{~mm}$ e facilidade de visualização. $(7,10)$ No entanto, um quarto a um terço das fístulas nunca maturam adequadamente. ${ }^{(11)}$ Estudo recen- te verificou que apenas $17,1 \%$ dos pacientes em hemodiálise utilizaram a fístula após dois meses e $54,7 \%$ após quatro meses de criação. ${ }^{(12)}$

A avaliação dessas mudanças deve envolver a observação e avaliação do acesso vascular por meio do exame físico; e a vigilância, que se refere à avaliação periódica utilizando testes que envolvem instrumentação especial, como uso do Ultrassom Doppler (USD) e angiografia. ${ }^{(10,13-15)}$ No geral, ambas avaliaçóes são complementares. ${ }^{(16-18)}$

A avaliação física do monitoramento de disfunção do acesso na fase de maturação, foco deste artigo, tem sido considerada conveniente, simples, econômica e de relevância. ${ }^{(16)}$ Estudo desenvolvido em uma unidade radiológica demonstrou que esta avaliação obteve altos valores de sensibilidade (82\%) para detecção de estenose, sendo o exame físico uma ferramenta útil, especialmente quando aplicado em centros que não dispóem da ultrassonografia para vigilância. ${ }^{(17)}$ Além disso, com menos limites de uso do que o USD, como custo e necessidade de operador habilitado. ${ }^{(9)}$

Revisão sistemática identificou o exame físico como ferramenta efetiva e acurada na detecção de disfunção na FAV, contudo, indicou sua inadequada realização. Concluiu que a assistência de enfermagem é mais próxima, regular e frequente aos pacientes com FAV, justificando a condução dessa 
avaliação pelos enfermeiros. ${ }^{(9)}$ Estudo com enfermeiros portugueses de hemodiálise de oito centros especializados apontou fragilidade relacionada às habilidades e aos conhecimentos para realizar essa avaliação física. ${ }^{(19)}$ Ademais, observam-se variaçôes nas recomendaçóes institucionais sobre elementos de avaliação da FAV com consequências sobre a delimitação das ações profissionais.

Diante das fragilidades apontadas, entende-se ser necessário o mapeamento das evidências disponíveis acerca dos elementos utilizados para a avaliação clínica e caracterizar as técnicas utilizadas pelos profissionais que a tem realizado, de forma a facilitar posteriores padronizaçóes para as práticas profissionais e produzir melhores resultados no acompanhamento da maturação da FAV.

O objetivo do estudo foi mapear as evidências de pesquisa sobre a avaliação clínica da maturação da fístula arteriovenosa em pacientes com doença renal crônica.

\section{Métodos}

Estudo do tipo Scoping Review, com revisão sistemática, orientada pela metodologia do Joanna Briggs Institute (JBI) e Joanna Briggs Collaborating Centres. (20,21) A seleção dessa estratégia de revisão baseou-se em suas finalidades de mapear o corpo de conhecimento em um tópico e incorporar uma amplitude de desenhos de estudo e metodologias de pesquisa. ${ }^{(22)}$

As etapas realizadas foram: Definiçáo e alinhamento dos objetivos e questóes da revisão; Desenvolvimento e alinhamento dos critérios de inclusão com objetivos e questôes; Descrição da abordagem planejada para seleção, extração e mapeamento das evidências de pesquisa; Busca pelas evidências; Seleção das evidências; Extração das evidências; Mapeamento das evidências; Sumarização das evidências em relação ao objetivo e a questão; Consulta a cientistas da informação, bibliotecários e/ou especialistas.

A pergunta da pesquisa usou a estratégia PCC, sendo Population - adultos com insuficiência renal crônica, Concept- elementos para avaliação clínica da maturação da FAV para hemodiálise e Context- período pós-operatório de confecção da FAV, em qualquer cenário hospitalar. Assim construída: "Quais são os elementos da maturação a serem avaliados clinicamente na FAV para hemodiálise no período pós-operatório de sua confecção em pacientes adultos com insuficiência renal crônica?”

Foram critérios de inclusão: estudos realizados com pacientes maiores de 18 anos, com DRC pré-dialítica ou em hemodiálise, submetidos à cirurgia da FAV; texto completo disponível; e nos idiomas inglês, espanhol ou português. Foram excluídas publicações não relacionadas à fase pós-operatória, relacionadas a cuidados com próteses/enxertos arteriovenosos e que não abordavam a avaliação clínica na fase de maturação.

A busca ocorreu em outubro e novembro de 2019, nas bases de dados: JBI; Cochrane; todas as bases de dados da Biblioteca Virtual em Saúde; PubMed e CINAHL. As evidências da gray literature foram buscadas no Catálogo de Teses e Dissertaçóes da CAPES, documentos em sites de órgãos oficiais, diretrizes e manuais de instituiçóes internacionais e nacionais, e livros. Não houve limitação temporal para busca. Utilizou-se os descritores em Ciências da Saúde (DECS) e Medical Subject Headings (MeSH) ligados à pergunta da pesquisa (Quadro 1).

Quadro 1. Estratégia de busca e descritores utilizados

\begin{tabular}{|c|c|c|}
\hline Estratégia “PCC" & Descritores do DECS & MESH terms \\
\hline $\begin{array}{l}\text { POPULAÇÃO: Pacientes } \\
\text { adultos com insuficiência } \\
\text { renal crônica }\end{array}$ & $\begin{array}{l}\text { Adulto OR Adulto jovem OR } \\
\text { Pessoas de meia idade AND } \\
\text { Insuficiência renal }\end{array}$ & $\begin{array}{l}\text { Adult OR Young adult OR } \\
\text { Middle aged AND Chronic } \\
\text { kidney failure }\end{array}$ \\
\hline $\begin{array}{l}\text { CONCEITO: Indicadores } \\
\text { para avaliação clínica da } \\
\text { maturação da FAV para } \\
\text { hemodiálise }\end{array}$ & $\begin{array}{l}\text { Fístula arteriovenosa AND } \\
\text { Avaliação em saúde OR } \\
\text { Avaliação em enfermagem } \\
\text { OR } \\
\text { Exame Físico OR Anamnese } \\
\text { AND Diálise Renal }\end{array}$ & $\begin{array}{l}\text { Arteriovenous fistula AND } \\
\text { Health Care Evaluation } \\
\text { Mechanisms OR Nursing } \\
\text { Assessment OR Physical } \\
\text { Examination OR Medical } \\
\text { History Taking AND Renal } \\
\text { Dialysis }\end{array}$ \\
\hline $\begin{array}{l}\text { CONTEXTO: Período pós- } \\
\text { operatório de confecção da } \\
\text { FAV, em qualquer cenário } \\
\text { hospitalar }\end{array}$ & $\begin{array}{l}\text { Período pós-operatório OR } \\
\text { Cuidados pós-operatórios }\end{array}$ & $\begin{array}{l}\text { Postoperative period OR } \\
\text { Postoperative Care }\end{array}$ \\
\hline
\end{tabular}

As citações identificadas foram coletadas e carregadas no Mendeley ${ }^{\oplus}$ e duplicadas removidas. A leitura exploratória de títulos e resumos foi realizada por pares de revisores de forma independente, julgando os estudos que tinham relaçôes com a pergunta de pesquisa e atendiam critérios de inclusão de idioma e disponibilidade do texto completo. As discordâncias foram resolvidas por consensualização entre pares ou pela avaliação de um terceiro revisor, 
quando mantida a discordância. Então, os estudos pré-selecionados foram lidos na íntegra para avaliação do conteúdo quanto à sua contribuição na compreensão do fenômeno estudado e posterior síntese de dados. Posteriormente, a consulta às listas de referência dos artigos em busca de estudos adicionais resultou na inclusão de mais oito publicaçôes. Todas as pesquisas, decisóes e etapas foram documentadas e arquivadas pelo revisor principal.

A busca e seleção dos estudos seguiu as recomendaçóes do JBI em relação a apresentação dos resultados com checklist adaptado do Preferred Reporting Items for Systematic Reviews and MetaAnalyses (PRISMA). ${ }^{(20)}$

Do corpus final dos estudos incluídos, foram extraídos os dados com formulário específico para mapeamento do título do artigo; autoria; ano de publicação; país de origem; objetivo; população do estudo e tamanho da amostra (quando aplicável); métodos; desenho de estudo; profissional responsável pela avaliação clínica; duração da avaliação (se aplicável); e técnicas de avaliação. Posteriormente foram inseridos em planilha Excel $^{\oplus}$, da qual procedeu-se a caracterização dos estudos e agrupamento, síntese e descrição dos resultados a partir da questão de pesquisa.

\section{Resultados}

Foram identificadas 1954 publicaçóes, dos quais 38 compuseram a amostra final. No fluxo do processo de busca (Figura 1), inicialmente foram encontradas 1940 correspondências. Outras quatorze foram adicionadas a partir de referências $(n=8)$ e literatura cinza $(n=6)$, removendo-se as duplicadas $(n=4)$, obteve-se 1950 estudos para leitura dos títulos e resumos. Por não responderem à pergunta de pesquisa, 1831 foram excluídos. Os textos completos dos 119 restantes foram avaliados quanto à elegibilidade, e 81 foram excluídos. Trinta e oito estudos foram incluídos no processo final de extração e análise de dados.

O quadro 2 apresenta as publicaçóes selecionadas conforme autoria/ano, título, desenho de estudo, objetivo, população/amostra e país de origem. Conforme observado, foram encontrados estudos publicados nos últimos 20 anos, entre 1998 e 2018. Dos tipos de publicaçóes, 29 são artigos (76,3\%), seis diretrizes clínicas (15,8\%), dois manuais (5,3\%), além de uma fonte de livro (2,6\%). A maioria das publicaçóes foi internacional $(n=36 ; 94,7 \%)$, com prevalência de publicaçóes dos Estados Unidos da América (EUA) (n=14; 36,8\%).

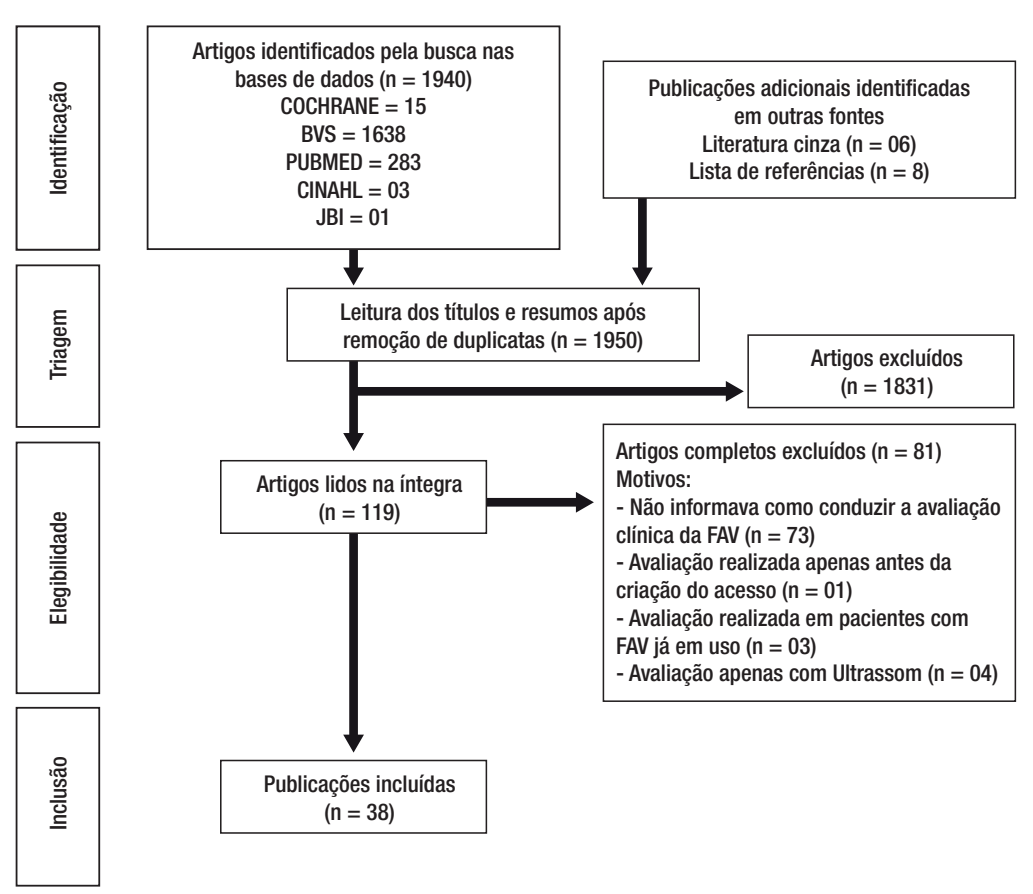

Figura 1. Fluxograma PRISMA do processo de busca na literatura 
Quadro 2. Descrição das publicações quanto à autoria e ano de publicação, título, desenho de estudo e país

\begin{tabular}{|c|c|c|c|c|c|}
\hline Autoria/ano & Título & $\begin{array}{l}\text { Desenho de } \\
\text { estudo }\end{array}$ & Objetivo & $\begin{array}{l}\text { População/ } \\
\text { amostra }\end{array}$ & $\begin{array}{l}\text { País de } \\
\text { origem }\end{array}$ \\
\hline $\begin{array}{l}\text { Schmidli et al. }{ }^{(10)} \\
2018\end{array}$ & $\begin{array}{l}\text { Vascular Access: } 2018 \text { Clinical Practice } \\
\text { Guidelines of the European Society for } \\
\text { Vascular Surgery }\end{array}$ & Revisão & $\begin{array}{l}\text { Estabelecer diretrizes de prática clínica para cirurgiões } \\
\text { e médicos envolvidos no atendimento de pacientes em } \\
\text { hemodiálise e com acesso vascular. }\end{array}$ & Não se aplica. & Suíça \\
\hline $\begin{array}{l}\text { National Kidney } \\
\text { Foundation }{ }^{(14)} \\
2006\end{array}$ & $\begin{array}{l}\text { Clinical Practice Guidelines and Clinical } \\
\text { Practice Recommendations }\end{array}$ & Revisão & $\begin{array}{l}\text { Divulgar as diretrizes e recomendações clínicas para } \\
\text { acesso vascular emitidas pelo grupo de trabalho da } \\
\text { National Kidney Foundation. }\end{array}$ & Não se aplica. & EUA \\
\hline $\begin{array}{l}\text { Ibeas et al. }{ }^{(15)} \\
2017\end{array}$ & $\begin{array}{l}\text { Guía Clínica Española del Acceso } \\
\text { Vascular para Hemodiálisis }\end{array}$ & Revisão & $\begin{array}{l}\text { Revisar as evidências da literatura e estabelecer } \\
\text { recomendações no manejo do acesso vascular para } \\
\text { hemodiálise que facilitem a tomada de decisões na prática } \\
\text { clínica. }\end{array}$ & Não se aplica. & Espanha \\
\hline $\begin{array}{l}\text { Beathard }{ }^{(23)} \\
1998\end{array}$ & $\begin{array}{l}\text { Physical examination of the dialysis } \\
\text { vascular access }\end{array}$ & Teorização & $\begin{array}{l}\text { Revisar os aspectos envolvidos no exame físico do acesso } \\
\text { vascular para hemodiálise. }\end{array}$ & Não se aplica. & EUA \\
\hline $\begin{array}{l}\text { Coentrão(24) } \\
2012\end{array}$ & $\begin{array}{l}\text { Physical examination of dysfunctional } \\
\text { arteriovenous fistulae by non- } \\
\text { interventionalist: a skill worth teaching }\end{array}$ & $\begin{array}{l}\text { Prospectivo } \\
\text { observacional }\end{array}$ & $\begin{array}{l}\text { Analisar a acurácia do exame físico na avaliação } \\
\text { de disfunção da FAV por não intervencionistas em } \\
\text { comparação com a angiografia. }\end{array}$ & $\begin{array}{l}177 \text { pacientes com disfunção } \\
\text { na FAV. }\end{array}$ & Portugal \\
\hline $\begin{array}{l}\text { McLafferty } \\
2007\end{array}$ & $\begin{array}{l}\text { Outcome of a comprehensive follow- } \\
\text { up program to enhance maturation of } \\
\text { autogenous arteriovenous hemodialysis } \\
\text { access }\end{array}$ & $\begin{array}{l}\text { Coorte } \\
\text { Prospectivo }\end{array}$ & $\begin{array}{l}\text { Analisar o resultado de um programa abrangente de } \\
\text { acompanhamento do acesso autógeno para hemodiálise } \\
\text { quando realizado pelo cirurgião vascular. }\end{array}$ & $\begin{array}{l}113 \text { pacientes submetidos à } \\
\text { cirurgia de construção de FAV. }\end{array}$ & EUA \\
\hline $\begin{array}{l}\text { Sidawy } \\
2008\end{array}$ & $\begin{array}{l}\text { The Society for Vascular Surgery: } \\
\text { Clinical practice guidelines for the } \\
\text { surgical placement and maintenance of } \\
\text { arteriovenous hemodialysis access }\end{array}$ & Revisão & $\begin{array}{l}\text { Desenvolver diretrizes práticas na colocação e } \\
\text { manutenção de acesso arteriovenoso, com o objetivo de } \\
\text { maximizar a porcentagem e a funcionalidade de acessos } \\
\text { arteriovenosos autógenos que são confeccionados. }\end{array}$ & Não se aplica. & EUA \\
\hline $\begin{array}{l}\text { Leivaditis }^{(27)} \\
2014\end{array}$ & $\begin{array}{l}\text { Vascular access for hemodialysis: } \\
\text { postoperative evaluation and function } \\
\text { monitoring }\end{array}$ & Revisão & $\begin{array}{l}\text { Revisar aspectos envolvidos na monitoração pós- } \\
\text { operatória do acesso vascular para hemodiálise. }\end{array}$ & Não se aplica. & Grécia \\
\hline $\begin{array}{l}\text { Ferring }^{(28)} \\
2014\end{array}$ & $\begin{array}{l}\text { Accuracy of early postoperative } \\
\text { clinical and ultrasound examination of } \\
\text { arteriovenous fistulae to predict dialysis } \\
\text { use }\end{array}$ & Randomizado & $\begin{array}{l}\text { Avaliar a acurácia da avaliação clínica e realização do } \\
\text { ultrassom precoce na predição de funcionalidade da FAV. }\end{array}$ & $\begin{array}{l}208 \text { pacientes submetidos a } \\
\text { cirurgia de construção de FAV. }\end{array}$ & Inglaterra \\
\hline $\begin{array}{l}\text { Robbin }^{(29)} \\
2002\end{array}$ & $\begin{array}{l}\text { Hemodialysis arteriovenous fistula } \\
\text { maturity: US evaluation }\end{array}$ & $\begin{array}{l}\text { Retrospectivo } \\
\text { comparativo }\end{array}$ & $\begin{array}{l}\text { Comparar critérios ultrassonográficos objetivos para a } \\
\text { maturação da FAV nativa com os resultados subsequentes } \\
\text { da fístula e avaliação clínica por enfermeiros experientes } \\
\text { em diálise. }\end{array}$ & $\begin{array}{l}69 \text { pacientes no pós-operatório } \\
\text { da cirurgia de construção de } \\
\text { FAV submetidos a avaliação por } \\
\text { ultrassom. }\end{array}$ & EUA \\
\hline $\begin{array}{l}\text { Leon }^{(30)} \\
2008\end{array}$ & $\begin{array}{l}\text { Physical examination of arteriovenous } \\
\text { fistula by a renal fellow: does it compare } \\
\text { favorably to an experienced intervention } \\
\text { a list? }\end{array}$ & $\begin{array}{l}\text { Prospectivo } \\
\text { Transversal }\end{array}$ & $\begin{array}{l}\text { Examinar a acurácia do exame físico em comparação com } \\
\text { a angiografia quando realizado por nefrologistas. }\end{array}$ & $\begin{array}{l}\text { Avaliação por nefrologistas de } \\
45 \text { casos de disfunção de FAV e } \\
\text { avaliação por intervencionistas } \\
\text { de } 142 \text { pacientes com disfunção } \\
\text { na fistula. }\end{array}$ & EUA \\
\hline $\begin{array}{l}\text { Beaumont Hospital|31) } \\
2012\end{array}$ & $\begin{array}{l}\text { Guidelines on the Management of } \\
\text { Arteriovenous Fistula and Grafts }\end{array}$ & Revisão & $\begin{array}{l}\text { Maximizar a eficiência e segurança do paciente renal com } \\
\text { FAV e enxerto arteriovenoso. }\end{array}$ & Não se aplica. & Irlanda \\
\hline $\begin{array}{l}\text { European Dialysis } \\
\text { and Transplant } \\
\text { Nurses Association/ } \\
\text { Eruropean Renal Care } \\
\text { Association }{ }^{(32)} \\
2015\end{array}$ & $\begin{array}{l}\text { Manual de boas práticas de enfermagem } \\
\text { para a fístula arteriovenosa }\end{array}$ & Teorização & $\begin{array}{l}\text { Análise de capítulo cujo objetivo é descrever aspectos } \\
\text { relacionados a FAV, incluindo avaliação pós-operatótia. }\end{array}$ & Não se aplica. & Portugal \\
\hline $\begin{array}{l}\text { Thomas }^{(33)} \\
2013\end{array}$ & Renal Nursing & Teorização & $\begin{array}{l}\text { Capítulo de livro descrevendo aspectos envolvidos na } \\
\text { avaliação clínica da FAV. }\end{array}$ & Não se aplica. & Inglaterra \\
\hline $\begin{array}{l}\text { Mattiazzi }^{(34)} \\
1999\end{array}$ & Evaluación clínica del acceso vascular & Teorização & $\begin{array}{l}\text { Abordar a importância da avaliação clínica da FAV } \\
\text { utilizando o exame físico. }\end{array}$ & Não se aplica. & Argentina \\
\hline $\begin{array}{l}\text { Beathard(35) } \\
2005\end{array}$ & $\begin{array}{l}\text { An algorithm for the physical examination } \\
\text { of early fistula failure }\end{array}$ & Teorização & $\begin{array}{l}\text { Revisar e definir uma abordagem para avaliação precoce } \\
\text { da falha na maturação da FAV utilizando o exame físico. }\end{array}$ & Não se aplica. & EUA \\
\hline $\begin{array}{l}\operatorname{Kian}^{(36)} \\
2005\end{array}$ & $\begin{array}{l}\text { The new arteriovenous fistula: the need } \\
\text { for earlier evaluation and intervention }\end{array}$ & Revisão & $\begin{array}{l}\text { Revisar a importância da avaliação e intervenção precoce } \\
\text { na FAV em maturação. }\end{array}$ & Não se aplica. & EUA \\
\hline $\begin{array}{l}\text { Asif }(37) \\
2006\end{array}$ & $\begin{array}{l}\text { Early arteriovenous fistula failure:a logical } \\
\text { proposal for when and how to intervene }\end{array}$ & Revisão & $\begin{array}{l}\text { Descrever o entendimento atual da maturação da fístula e } \\
\text { falha precoce e revisar as alterações no fluxo sanguíneo } \\
\text { e no tamanho que ocorrem em uma FAV recém-criada e } \\
\text { apresentar informações sobre quando e como intervir para } \\
\text { identificar e recuperar fístulas com falha precoce. }\end{array}$ & Não se aplica. & EUA \\
\hline $\begin{array}{l}\text { Ohira(38) } \\
2006\end{array}$ & $\begin{array}{l}2005 \text { Japanese Society for Dialysis } \\
\text { Therapy Guidelines for vascular access } \\
\text { construction and repair for chronic } \\
\text { hemodialysis }\end{array}$ & Revisão & $\begin{array}{l}\text { Instituir as diretrizes japonesas para construção e reparo } \\
\text { de acesso vascular para hemodiálise, padronizando os } \\
\text { procedimentos relacionados ao acesso vascular. }\end{array}$ & Não se aplica. & Japão \\
\hline $\begin{array}{l}\text { Jiménez Almonacid(39) } \\
2007\end{array}$ & $\begin{array}{l}\text { Definición de procesos e indicadores } \\
\text { para la gestión de accesos vasculares } \\
\text { para hemodiálises }\end{array}$ & Metodológico & $\begin{array}{l}\text { Definir processos para o manejo do acesso vascular para } \\
\text { hemodiálise e avaliar esses processos. }\end{array}$ & $\begin{array}{l}\text { Prontuários de pacientes com } \\
\text { acesso vascular para hemodiálise }\end{array}$ & Espanha \\
\hline
\end{tabular}

Continua... 


\begin{tabular}{|c|c|c|c|c|c|}
\hline Autoria/ano & Título & $\begin{array}{l}\text { Desenho de } \\
\text { estudo }\end{array}$ & Objetivo & $\begin{array}{l}\text { População/ } \\
\text { amostra }\end{array}$ & $\begin{array}{l}\text { Pais de } \\
\text { origem }\end{array}$ \\
\hline $\begin{array}{l}\text { Levine } e^{(40)} \\
2008\end{array}$ & $\begin{array}{l}\text { A challenge for nephrologists -increasing } \\
\text { fistula maturation rates, reducing fistula } \\
\text { maturation time, and decreasing dialysis } \\
\text { catheter prevalence in the United States }\end{array}$ & Revisão & $\begin{array}{l}\text { Refletir sobre a importância do estabelecimento de FAV } \\
\text { para hemodiálise. }\end{array}$ & Não se aplica. & EUA \\
\hline $\begin{array}{l}\text { McCann }^{(41)} \\
2008\end{array}$ & $\begin{array}{l}\text { Vascular Access Management 1:An } \\
\text { overview }\end{array}$ & Revisão & $\begin{array}{l}\text { Descrever os aspectos envolvidos no manejo do acesso } \\
\text { vascular para hemodiálise. }\end{array}$ & Não se aplica. & Irlanda \\
\hline $\begin{array}{l}\text { Nassar } r^{(42)} \\
2008\end{array}$ & $\begin{array}{l}\text { Endovascular Management of the } \\
\text { "Failing to Mature" Arteriovenous Fistula }\end{array}$ & Revisão & $\begin{array}{l}\text { Descrever as condutas diante da falha na maturação da } \\
\text { FAV. }\end{array}$ & Não se aplica. & EUA \\
\hline $\begin{array}{l}\text { Malovrh }^{(43)} \\
2010\end{array}$ & $\begin{array}{l}\text { Non-matured arteriovenous fistulae for } \\
\text { haemodialysis: diagnosis endovascular } \\
\text { and surgery treatment }\end{array}$ & Revisão & $\begin{array}{l}\text { Revisar o processo de maturação da FAV e apresentar } \\
\text { informações sobre como obter uma fístula madura. }\end{array}$ & Não se aplica. & Eslovênia \\
\hline $\begin{array}{l}\text { Neves Junior(44) } \\
2011\end{array}$ & $\begin{array}{l}\text { Avaliação da perviedade precoce das } \\
\text { fístulas arteriovenosas para hemodiálise }\end{array}$ & \begin{tabular}{|l|} 
Prospectivo \\
não- \\
randomizado \\
\end{tabular} & $\begin{array}{l}\text { Avaliar a perviedade precoce das FAV, identificando os } \\
\text { fatores relacionados ao insucesso destas. }\end{array}$ & $\begin{array}{l}31 \text { pacientes submetidos à } \\
\text { confecção de fistula arteriovenosa. }\end{array}$ & Brasil \\
\hline $\begin{array}{l}\text { Ahmed(45) } \\
2012\end{array}$ & $\begin{array}{l}\text { Outcomes of arteriovenous fistula for } \\
\text { hemodialysis in Sudanese patients: } \\
\text { single-center experience. }\end{array}$ & $\begin{array}{l}\text { Prospectivo } \\
\text { randomizado }\end{array}$ & $\begin{array}{l}\text { Descrever a experiência de um centro de diálise na } \\
\text { criação de FAV e avaliar a taxa de sucesso e complicações } \\
\text { comuns. }\end{array}$ & 73 pacientes em hemodiálise. & Sudão \\
\hline $\begin{array}{l}\operatorname{Rose}^{(46)} \\
2013\end{array}$ & Hemodialysis Access & Revisão & $\begin{array}{l}\text { Descrever fatores relacionados ao acesso vascular para } \\
\text { hemodiálise. }\end{array}$ & Não se aplica. & EUA \\
\hline $\begin{array}{l}\text { Salimi } 447 \\
2013\end{array}$ & $\begin{array}{l}\text { Assessment of effects of upper extremity } \\
\text { exercise with arm } \\
\text { tourniquet on maturity of arteriovenous } \\
\text { fistula in hemodialysis patients }\end{array}$ & Randomizado & $\begin{array}{l}\text { Investigar os efeitos do exercício manual usando um } \\
\text { torniquete na FAV em maturação em pacientes em estágio } \\
\text { renal terminal. }\end{array}$ & $\begin{array}{l}50 \text { pacientes submetidos à } \\
\text { construção da FAV. }\end{array}$ & Irã \\
\hline $\begin{array}{l}\text { Sousa(48) } \\
2013\end{array}$ & $\begin{array}{l}\text { Physical examination: How to examine } \\
\text { the arm with arteriovenous fistula }\end{array}$ & Revisão & $\begin{array}{l}\text { Reunir e sistematizar o conhecimento sobre aspectos do } \\
\text { exame físico que permitem a identificação } \\
\text { das complicações da FAV. }\end{array}$ & Não se aplica. & Portugal \\
\hline $\begin{array}{l}\text { Huang }(49) \\
2014\end{array}$ & $\begin{array}{l}\text { Compliance with Surgical Follow-up Does } \\
\text { Not Influence Fistula Maturation in a } \\
\text { County Hospital Population }\end{array}$ & Prospectivo & $\begin{array}{l}\text { Examinar as taxas de acompanhamento e as taxas de } \\
\text { maturação após a cirurgia de criação de fístula em um } \\
\text { hospital municipal com uma população indigente. }\end{array}$ & $\begin{array}{l}203 \text { pacientes submetidos a } \\
\text { cirurgia de construção de FAV. }\end{array}$ & EUA \\
\hline $\begin{array}{l}\text { Malovrh(50) } \\
2014\end{array}$ & $\begin{array}{l}\text { Postoperative assessment of vascular } \\
\text { access }\end{array}$ & Revisão & $\begin{array}{l}\text { Revisar e estabelecer critérios para avaliação pós- } \\
\text { operatória do acesso vascular. }\end{array}$ & Não se aplica. & Eslovênia \\
\hline $\begin{array}{l}\text { López Alonso } \\
2015\end{array}$ & $\begin{array}{l}\text { Influencia del ejercicio físico en el } \\
\text { desarrollo de fístulas arteriovenosas } \\
\text { nativas }\end{array}$ & $\begin{array}{l}\text { Prospectivo } \\
\text { randomizado }\end{array}$ & $\begin{array}{l}\text { Avaliar a influência de uma enfermeira nefrologista } \\
\text { experiente em um programa controlado de exercício físico } \\
\text { pós-operatório na maturação da FAV nativa no mês de sua } \\
\text { implantação. }\end{array}$ & $\begin{array}{l}68 \text { pacientes em pós-operatório de } \\
\text { cirurgia de construção de FAV. }\end{array}$ & Espanha \\
\hline $\begin{array}{l}\text { Cheng }{ }^{(52)} \\
2015\end{array}$ & $\begin{array}{l}\text { The reasons for the failure of the primary } \\
\text { arteriovenous fistula surgery in patients } \\
\text { with end-stage renal disease }\end{array}$ & $\begin{array}{l}\text { Prospectivo } \\
\text { transversal }\end{array}$ & $\begin{array}{l}\text { Analisar as razões para a falha primária da cirurgia de FAV } \\
\text { e explorar as medidas preventivas. }\end{array}$ & $\begin{array}{l}819 \text { pacientes submetidos a } \\
\text { cirurgia de criação de FAV. }\end{array}$ & China \\
\hline $\begin{array}{l}\text { Mufty } \\
2015\end{array}$ & $\begin{array}{l}\text { Proactive surveillance approach to } \\
\text { guarantee a functional } \\
\text { arteriovenous fistula at first dialysis is } \\
\text { worth }\end{array}$ & $\begin{array}{l}\text { Coorte } \\
\text { Prospectivo }\end{array}$ & $\begin{array}{l}\text { Avaliar o impacto de um programa de vigilância proativa } \\
\text { na taxa de acesso funcional no momento da primeira } \\
\text { diálise. }\end{array}$ & $\begin{array}{l}164 \text { pacientes submetidos a } \\
\text { cirurgia de construção de FAV. }\end{array}$ & Bélgica \\
\hline $\begin{array}{l}\text { Rosenberg(54) } \\
2015\end{array}$ & $\begin{array}{l}\text { Prediction of Arteriovenous Fistula } \\
\text { Dysfunction: Can it be Taught? }\end{array}$ & $\begin{array}{l}\text { Prospectivo } \\
\text { transversal }\end{array}$ & $\begin{array}{l}\text { Avaliar se é possível o ensino do exame físico a } \\
\text { estudantes e verificar acurácia do exame feito por eles na } \\
\text { detecção de estenose quando comparado ao exame físico } \\
\text { feito por profissionais intervencionistas. }\end{array}$ & $\begin{array}{l}49 \text { pacientes com suspeita de } \\
\text { disfunção no acesso. }\end{array}$ & EUA \\
\hline $\begin{array}{l}\text { Pereira(55) } \\
2016\end{array}$ & $\begin{array}{l}\text { Avaliação do tempo de maturação das } \\
\text { fístulas rádio-cefálicas para hemodiálise }\end{array}$ & $\begin{array}{l}\text { Observacional } \\
\text { retrospectivo }\end{array}$ & $\begin{array}{l}\text { Avaliar se o tempo de maturação das FAV rádio-cefálicas } \\
\text { é influenciado pelo gênero e idade dos pacientes ou pela } \\
\text { causa da insuficiência renal. }\end{array}$ & $\begin{array}{l}66 \text { prontuários de pacientes } \\
\text { submetidos à FAV rádio-cefálica }\end{array}$ & Brasil \\
\hline $\begin{array}{l}\text { Martinez } z^{(56)} \\
2017\end{array}$ & $\begin{array}{l}\text { Neuromuscular electro stimulation: a } \\
\text { new therapeutic option to improve radio } \\
\text { cephalic arteriovenous fistula maturation } \\
\text { in end stage chronic kidney disease } \\
\text { patients }\end{array}$ & $\begin{array}{l}\text { Prospectivo } \\
\text { caso-controle }\end{array}$ & $\begin{array}{l}\text { Avaliar a utilidade de um programa neuromuscular de } \\
\text { eletroestimulação na maturação da FAV. }\end{array}$ & $\begin{array}{l}36 \text { pacientes submetidos à criação } \\
\text { da FAV. }\end{array}$ & Espanha \\
\hline $\begin{array}{l}\text { Rodrigues }^{(57)} \\
2018\end{array}$ & $\begin{array}{l}\text { Avaliação de variáveis associadas à } \\
\text { permeabilidade de fístulas arteriovenosas } \\
\text { criadas por um nefrologista para fins de } \\
\text { hemodiálise }\end{array}$ & $\begin{array}{l}\text { Coorte } \\
\text { retrospectivo }\end{array}$ & $\begin{array}{l}\text { Avaliar a taxa de sucesso da FAV criada por um } \\
\text { nefrologista e identificar variáveis clínicas, laboratoriais e } \\
\text { demográficas que influenciam a patência da FAV. }\end{array}$ & $\begin{array}{l}101 \text { pacientes com doença renal } \\
\text { crônica. }\end{array}$ & Brasil \\
\hline
\end{tabular}

Os estudos apontaram que em relação aos profissionais responsáveis pela avaliação predominou o enfermeiro nefrologista ou equipe de enfermagem $(n=18 ; 47,4 \%)$, seguida do médico nefrologista, intervencionista ou cirurgião vascular $(n=16$;
$42,1 \%$ ), e por fim, profissional ou equipe especializada/capacitada para lidar especificamente com a FAV ( $\mathrm{n}=6 ; 15,8 \%)$.

O tempo de avaliação pós-cirúrgica da FAV variou desde as primeiras horas de pós-operatório até 
Quadro 3. Elementos clínicos de avaliação da maturação da fístula arteriovenosa

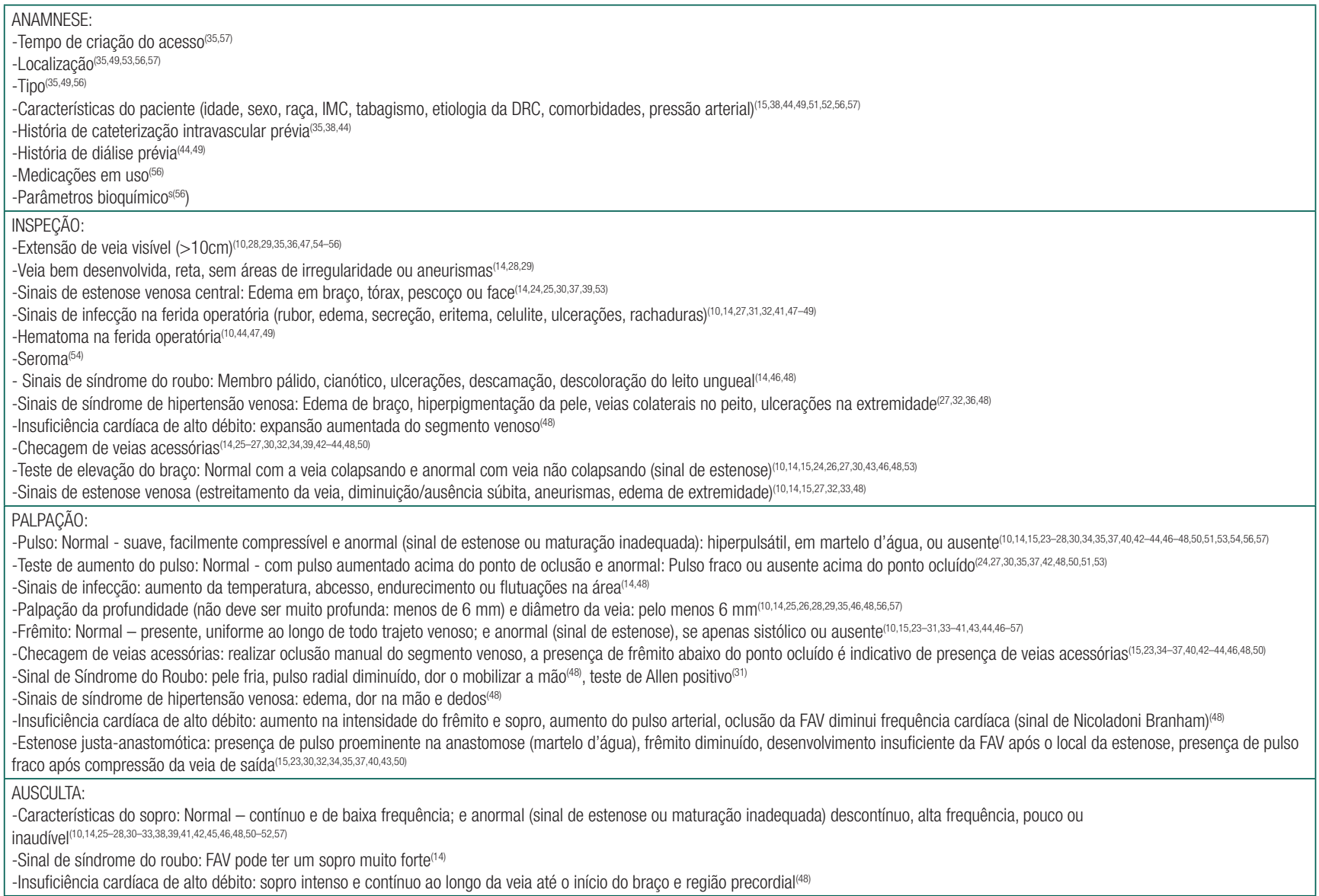

IMC - Índice de massa corporal; DRC - Doença Renal Crônica

um ano. Duas publicaçóes recomendaram a avaliação nas primeiras horas, inicialmente a cada meia hora, e depois com intervalos decrescentes até a alta. ${ }^{(33,41)} \mathrm{A}$ avaliação semanal pós-cirúrgica; ${ }^{(55)}$ no $1^{\circ}, 10^{\circ}$ e $30^{\circ}$ dias pós-operatório; ${ }^{(44)}$ ou no $1^{\circ}$ dia, $1^{\mathrm{a}}$ semana e na $4^{\mathrm{a}}$ semana, ${ }^{(45)}$; dentro das primeiras duas semanas; ${ }^{(38)}$ após duas semanas; ${ }^{(47,49)}$ após quatro semanas; ${ }^{(28,51)}$ dentro de seis semanas; ${ }^{(32)}$ de três a seis semanas ${ }^{(25)}$ foram outros padróes evidenciados. Contudo, a maioria indicou avaliação da maturação dentro de quatro até seis semanas. ${ }^{(10,14,15,26,31,35,37,43,52)}$

Ainda foram verificados estudos apontando tempos superiores a seis semanas: entre quatro e oito semanas; ${ }^{(56,57)}$ dentro de dois meses após o procedimento; ${ }^{(40)}$ até dois a três meses; ${ }^{(33)}$ dentro de quatro meses; ${ }^{(29)} \mathrm{em}$ seis meses; ${ }^{(54)}$ e outro que recomendou realizar a avaliação em duas semanas, seis semanas, três meses, seis meses e 12 meses do período pós-operatório. ${ }^{(53)}$

Dentre as técnicas de avaliação clínica, 23 estudos $(60,5 \%)$ recomendaram o exame físico e 15 (39,5\%) a combinação anamnese e exame físico. Os elementos clínicos sinalizados como necessários para avaliar a maturação estáo sintetizados no quadro 3 .

\section{Discussão}

Os resultados indicaram uma diversidade de elementos de avaliação para acompanhamento da maturação da FAV com predominância do exame físico como estratégia de avaliação.

A acurácia do exame físico na avaliação da maturação da FAV quando comparado ao Doppler ultrassonográfico ${ }^{(28,29)}$ converge para os achados de estudo com pacientes de uma unidade de radiologia, no qual o exame físico superou o USD para diagnosticar estenose (62\% versus $58 \%$ de detecção). ${ }^{(18)}$ De acordo com a Resolução COFEN no 358/2009, que dispóe sobre as etapas do Processo de Enfermagem, o enfermeiro deve aplicar o Processo de Enfermagem em 
todo ambiente que ocorre o cuidado de enfermagem, sendo o exame físico técnica usada na etapa de coleta de dados de Enfermagem, fornecendo indicadores clínicos de relevância para tomada de decisão.

Condiçôes particulares podem indicar necessidade de educação continuada de elementos ligados à semiotécnica, dentre eles a ausculta de sopros indicativos de estenose de vasos, o que pode exigir uso adequado do estetoscópio para correta interpretação dos sons verificados.

Quanto à anamnese, diferentes estudos apontaram para um foco em dados cirúrgicos e características do paciente referentes a dados sociodemográficos, clínicos e de estilo de vida. ${ }^{15,35,38,44,49,51-53,56,57)}$ A relevância desses dados pode ser constatada em estudo que analisou a associaçáo com as informaçóes dos pacientes que estabeleceram FAV ao longo do período de um ano. Verificou-se associação de pacientes com idade avançada, sexo feminino, negros, apresentando comorbidades (doença cardiovascular, doença arterial periférica, diabetes, pacientes internados ou necessitando de assistência), em diálise há mais de um ano e utilizando cateter ou enxerto arteriovenoso com menor taxa de sucesso na maturaçáo da FAV. ${ }^{(12)}$

Desta feita, os dados investigados na anamnese devem ser abrangentes e categorizados em: fatores humanos, como idade avançada e sexo; fatores relacionados a marcadores sanguíneos como distúrbios de coagulação, uso de anticoagulantes, dislipidemia, uremia e hipoalbuminemia; estilo de vida e comorbidades associadas, como por exemplo, diabetes, hipertensão, doença vascular periférica, tabagismo e obesidade, ${ }^{(4)}$ além de outros fatores mais diretamente ligados à fístula, como disfunção endotelial, diâmetro de veia e pacientes em diálise portando CVC. ${ }^{(58)}$

Identificou-se nas publicaçóes analisadas que a avaliação da FAV, por meio do exame físico, deve ser estruturada na inspeção, palpação e ausculta, que quando adequadamente combinadas, constituem parte fundamental no atendimento ao paciente com acesso vascular. Essa combinação produz informaçôes cruciais que orientam a detecção de problemas e realização em tempo hábil de intervenções que previnam complicaçóes graves, perda potencial do acesso e maus resultados. ${ }^{(59)}$
Elementos da inspeção foram destacados na maioria das publicações com dezenas de critérios indicados. As informaçóes desta etapa podem ser importantes na detecção de problemas como infecçôes, aneurismas, sangramentos, estenoses de segmento venoso e central e síndrome do roubo. ${ }^{(16,59)}$

A palpação foi a única etapa do exame físico presente em todas as publicaçóes, com a maioria dessas indicando que as avaliaçóes por meio da palpação devem ser direcionadas a interpretar dados de fluxo sanguíneo, alteraçóes de diâmetro como estenose da luz do vaso e verificação de sinais de infecção. A ausculta também foi descrita em 21 publicaçóes analisadas (55,2\%), voltada à avaliação das características do sopro. Essas duas etapas são úteis para confirmar os achados da inspeção e fornecem informaçôes adicionais, sendo o frêmito, pulso e sopro elementos importantes para achados de problemas relacionados ao fluxo sanguíneo no acesso. ${ }^{(16)}$

Quanto ao período para avaliação pós-operatória, a variação abrangente indica que não há uma definição clara do período de avaliação. Esta falta de convergência sobre a regularidade do monitoramento do acesso pode ter como consequência postergar a identificação de problemas na maturação, alguns deles às vezes já irreversíveis. Isso pode requerer iniciativas de novas investigaçôes com desenhos de síntese, como por exemplo metanálises. Estudo multicêntrico para investigar o desenvolvimento da FAV ao longo de seis semanas, verificou que em um dia a FAV já atinge $50 \%$ do fluxo sanguíneo adequado, e em duas semanas já alcança o diâmetro e fluxo esperado, sugerindo que a avaliação pós-operatória se inicie tão logo a FAV seja estabelecida, para identificação precoce de problemas de maturação, possibilitando o planejamento de alternativas para estabelecer o acesso, diminuindo a dependência do CVC. ${ }^{(60)}$

Um centro de diálise na Holanda avaliou os efeitos de um protocolo de avaliaçôes pré e pós-operatórias da maturação da FAV nas taxas de sucesso na maturação do acesso por meio de um estudo caso-controle. No grupo um, foram realizadas 72 cirurgias de FAV, sem implementação do protocolo estabelecido. No grupo dois, foram realizadas 74 cirurgias, com implantaçáo do protocolo proposto de avaliação pós-operatória: primeiramente procedeu-se com retorno do paciente para exame do acesso, remoção da sutura e educação 
uma semana após o procedimento; posteriormente, o paciente era submetido ao exame físico para investigação de sinais de trombose ou falha na maturação durante visitas na unidade três vezes na semana. $\mathrm{O}$ estudo demonstrou que com o acompanhamento realizado no grupo dois, houve aumento na taxa de sucesso dos acessos, sendo alcançadas taxas de permeabilidade primárias e secundárias significativamente mais altas, com aumento na patência primária de $36 \%$ para $49 \%$ e na secundária de $47 \%$ para $70 \%$, com diminuição de intervençóes cirúrgicas. ${ }^{(61)}$ Os resultados sugerem que mais estudos devem ser conduzidos para confirmar a hipótese de que a frequência de avaliação melhora o sucesso e duração dos acessos.

Estudo desenvolvido em um centro de diálise americano instituiu protocolo de conversão rápida de CVC para FAV, com avaliação duas semanas após a criação do acesso, nova avaliação após quatro semanas, e se a FAV não apresentasse sinais clínicos de maturação, um ultrassom era realizado no mesmo dia, se possível. A avaliação prosseguia até a FAV ser utilizada corretamente, e como resultado $99 \%$ dos cateteres foram convertidos em acesso arteriovenoso. ${ }^{(62)}$

Em relação ao profissional responsável pela avaliação clínica, o enfermeiro foi o mais citado nos estudos. Os enfermeiros em diálise mantém contato regular e frequente com pacientes com FAV, logo, são os profissionais apropriados para execução da avaliação utilizando o exame físico. ${ }^{(9)}$ Estudo desenvolvido com 212 pacientes em dois centros de diálise constatou que com estabelecimento de um protocolo implementado por enfermeiro, apoiado pela equipe multiprofissional, a taxa de uso de FAV aumentou de $45,0 \%$ para $64,3 \%$ e o uso de cateter diminuiu de $11,0 \%$ para $6,0 \%{ }^{(63)}$

$\mathrm{O}$ treinamento profissional é um componente importante para reconhecimento de potenciais incidentes na maturação e nas outras fases do processo. A experiência específica do profissional na realização da avaliação clínica é relevante, sendo que na ausência de um examinador experiente disponível, é preferível encaminhar o paciente para realizar USD. ${ }^{(16)}$ Apesar disso, o exame físico pode ser facilmente ensinado a profissionais de saúde num curto intervalo de tempo. ${ }^{(24,30,54)}$ Entretanto, anos de experiência profissional em geral não devem ser critérios presuntivos da qualidade do monitoramento, cabendo sempre execução de treinamentos focados nos elementos de relevância de avaliação, de forma a garantir um melhor resultado no monitoramento. ${ }^{(9,17)}$

Apesar do predomínio de avaliaçóes por profissionais de enfermagem, os estudos não reportaram uma perspectiva abrangente orientada pelo processo de enfermagem, teoria ou sistema de classificação de enfermagem. Entende-se que tal perspectiva possa fornecer suporte para o cuidado prestado e contribuir para o fortalecimento científico da enfermagem, devendo contemplar todos os estágios do processo, com intuito de promover a qualidade de vida do paciente renal crônico submetido à hemodiálise. ${ }^{(64)}$

Apontam-se como limitaçôes: o fato dos achados serem derivados de um grande número de estudos com mais de dez anos de publicação, o que nos remete a reflexão da necessidade de estudos atuais sobre a temática; a possibilidade de terem sido desconsiderados estudos publicados em outros idiomas; e também não ter sido avaliada a qualidade dos estudos como acontece nas revisóes sistemáticas. Contudo, tratando-se de uma revisão de mapeamento da produção, tais limites são incontornáveis, e ainda mais não chegam a produzir problemas de interpretação frente aos objetivos da pesquisa.

\section{Conclusão}

A presente revisão mapeou evidências que destacaram o profissional enfermeiro como mais frequente avaliador da FAV, entretanto ainda é demandada uma sistematização do exame físico por meio da inspeção, palpação e ausculta. Variados elementos de avaliaçáo ligados a permeabilidade do acesso e respostas vasculares foram apresentados. Também foi reportada a predominância em nível internacional da avaliação por enfermeiros, e a relevância do treinamento do profissional para uma interpretação acurada do amadurecimento. No entanto, destaca-se a ausência de uma abordagem de avaliação mais abrangente orientada pelo Processo e Teorias de Enfermagem e Linguagens Padronizadas o que pode indicar um novo campo de investigação e desenvolvimento do conhecimento na área. Dessa forma, estima-se que a melhor opção seja a incorporação de teorias, processo de enfermagem e terminolo- 
gias padronizadas da profissão, além da construção e validação de protocolos para acompanhamento pós-operatório de pacientes com FAV, de forma a garantir o foco disciplinar de enfermagem na avaliação. Ainda com isso, contribuir com a sobrevida do paciente, cujo acesso é vital para seu tratamento. Para o trabalho do enfermeiro, uma avaliação acurada, implica em atenção mais especializada e segura; para os gestores em minimização de custos do cuidado e dispersão da carga de trabalho dos profissionais, e possivelmente contribuição para o sistema de saúde, com redução das complicações decorrentes dos acessos para hemodiálise e decréscimo das pressôes sobre o sistema de cuidado como um todo.

\section{Referências}

1. Gesualdo GD, Zazzetta MS, Say KG, Orlandi FS. [Factors associated with the frailty of elderly people with chronic kidney disease on hemodialysis]. Cien Saude Colet. 2016;21(11):3493-8. Portuguese.

2. Arhuidese IJ, Orandi BJ, Nejim B, Malas M. Utilization, patency, and complications associated with vascular access for hemodialysis in the United States. J Vasc Surg. 2018;68(4):1166-74.

3. Hamadneh SA, Nueirat SA, Qadoomi' J, Shurrab M, Qunibi WY, Hamdan Z. Vascular access mortality and hospitalization among hemodialysis patients in Palestine. Saudi J Kidney Dis Transpl. 2018;29(1):120-6.

4. Soleymanian T, Sheikh V, Tareh F, Argani H, Ossareh S. Hemodialysis vascular access and clinical outcomes: an observational multicenter study. J Vasc Access. 2017;18(1):35-42.

5. Yeh LM, Chiu SY, Lai PC. The impact of vascular access types on hemodialysis patient long-term survival. Sci Rep. 2019;9(1):10708.

6. Al-Balas A, Lee T, Young CJ, Kepes JA, Barker-Finkel J, Allon M. The Clinical and Economic Effect of Vascular Access Selection in Patients Initiating Hemodialysis with a Catheter. J Am Soc Nephrol. 2017;28(12):3679-87.

7. Brown RS, Patibandla BK, Goldfarb-Rumyantzev AS. The survival benefit of "fistula first, catheter last" in hemodialysis is primarily due to patient factors. J Am Soc Nephrol. 2017;28(2):645-52.

8. Agarwal AK, Haddad NJ, Vachharajani TJ, Asif A. Innovations in vascular access for hemodialysis. Kidney Int. 2019;95(5):1053-63.

9. Jackson VE, Hurst $H$, Mitra S. Structured physical assessment of arteriovenous fistulae in haemodialysis access surveillance: A missed opportunity? J Vasc Access. 2018;19(3):221-9.

10. Schmidli J, Widmer MK, Basile C, de Donato G, Gallieni M, Gibbons CP, et al.; Esvs Guidelines Committee; Esvs Guidelines Reviewers. Editor's Choice - Vascular Access: 2018 Clinical Practice Guidelines of the European Society for Vascular Surgery (ESVS). Eur J Vasc Endovasc Surg. 2018;55(6):757-818.

11. Salimi F, Shahabi S, Talebzadeh H, Keshavarzian A, Pourfakharan M, Safaei M. Evaluation of diagnostic values of clinical assessment in determining the maturation of arteriovenous fistulas for satisfactory hemodialysis. Adv Biomed Res. 2017;6(1):18.
12. Woodside KJ, Bell S, Mukhopadhyay P, Repeck KJ, Robinson IT, Eckard $A R$, et al. Arteriovenous fistula maturation in prevalent hemodialysis patients in the United States: A National Study. Am J Kidney Dis. 2018;71(6):793-801.

13. Kukita K, Ohira S, Amano I, Naito H, Azuma N, Ikeda K, et al.; Vascular Access Construction and Repair for Chronic Hemodialysis Guideline Working Group, Japanese Society for Dialysis Therapy. 2011 update Japanese Society for Dialysis Therapy Guidelines of Vascular Access Construction and Repair for Chronic Hemodialysis. Ther Apher Dial. 2015;19 Suppl 1:1-39.

14. Vascular Access 2006 Work Group. Clinical practice guidelines for vascular access. Am J Kidney Dis. 2006;48 Suppl 1:S176-247.

15. Ibeas J, Roca-Tey R, Vallespín J, Moreno T, Moñux G, Martí-Monrós A, et al.; por el Grupo Español Multidisciplinar del Acceso Vascular (GEMAV). Guía clínica española del acceso vascular para hemodiálisis. Nefrologia. 2017;37 Suppl 1:1-191.

16. Abreo K, Amin BM, Abreo AP. Physical examination of the hemodialysis arteriovenous fistula to detect early dysfunction. J Vasc Access. 2019;20(1):7-11.

17. Maldonado-Cárceles AB, García-Medina J, Torres-Cantero AM. Performance of physical examination versus ultrasonography to detect stenosis in haemodialysis arteriovenous fistula. J Vasc Access. 2017;18(1):30-4.

18. Robbin ML, Greene T, Allon M, Dember LM, Imrey PB, Cheung AK, et al.; Hemodialysis fistula maturation study group. prediction of arteriovenous fistula clinical maturation from postoperative ultrasound measurements: findings from the hemodialysis fistula maturation study. J Am Soc Nephrol. 2018;29(11):2735-44.

19. Sousa CN, Teles P, Dias VF, Apóstolo JL, Figueiredo MH, Martins MM. Physical examination of arteriovenous fistula: the influence of professional experience in the detection of complications. Hemodial Int. 2014;18(3):695-9.

20. Joanna Briggs Institute (JBI). Methodology for JBI Scoping Reviews - Joanna Briggs 2015. [Internet]. Australia: JBl; c2015. [cited 2020 Jan 27]. Available from: http://joannabriggs.org/assets/docs/sumari/ Reviewers-Manual_Methodology-for-JBI-Scoping-Reviews_2015_ v2.pdf

21. Peters MD, Godfrey CM, Khalil H, Mclnerney P, Parker D, Soares CB. Guidance for conducting systematic scoping reviews. Int J Evid-Based Healthc. 2015;13(3):141-6.

22. Munn Z, Peters MD, Stern C, Tufanaru C, McArthur A, Aromataris E. Systematic review or scoping review? Guidance for authors when choosing between a systematic or scoping review approach. BMC Med Res Methodol. 2018;18(1):143.

23. Beathard GA. Physical examination of the dialysis vascular access. Semin Dial. 1998;11(4):231-6.

24. Coentrão L, Faria B, Pestana M. Physical examination of dysfunctional arteriovenous fistulae by non-interventionalists: a skill worth teaching. Nephrol Dial Transplant. 2012;27(5):1993-6.

25. McLafferty RB, Pryor RW 3rd, Johnson CM, Ramsey DE, Hodgson KJ. Outcome of a comprehensive follow-up program to enhance maturation of autogenous arteriovenous hemodialysis access. J Vasc Surg. 2007;45(5):981-5.

26. Sidawy AN, Spergel LM, Besarab A, Allon M, Jennings WC, Padberg FT Jr, et al.; Society for Vascular Surgery. The Society for Vascular Surgery: clinical practice guidelines for the surgical placement and maintenance of arteriovenous hemodialysis access. J Vasc Surg. 2008;48(5 Suppl):2S-25S. 
27. Leivaditis K, Panagoutsos S, Roumeliotis A, Liakopoulos V, Vargemezis V. Vascular access for hemodialysis: postoperative evaluation and function monitoring. Int Urol Nephrol. 2014;46(2):403-9.

28. Ferring $M$, Henderson J, Wilmink T. Accuracy of early postoperative clinical and ultrasound examination of arteriovenous fistulae to predict dialysis use. J Vasc Access. 2014;15(4):291-7.

29. Robbin ML, Chamberlain NE, Lockhart ME, Gallichio MH, Young CJ, Deierhoi $\mathrm{MH}$, et al. Hemodialysis arteriovenous fistula maturity: US evaluation. Radiology. 2002;225(1):59-64.

30. Leon C, Asif A. Physical examination of arteriovenous fistulae by a renal fellow: does it compare favorably to an experienced interventionalist? Semin Dial. 2008;21(6):557-60.

31. George D. Beaumont Hospital. Guidelines on the management of arterio venous fistula and grafts [Internet]. Dublin: Beaumont Hospital; 2012. [cited 2020 Jun 16]. Available from: http://www.beaumont.ie/ media/AVFguidelinefinalcopy1.pdf

32. Parosotto ME, Pancirova J, editores. Canulação e cuidado do acesso vascular. Manual de boas práticas de enfermagem para a fístula arteriovenosa. 2a ed. Madrid: Tomás Hermannos; 2015.

33. Thomas N. Renal rursing. 4th ed. England: Wiley-Blackwell; 2013.

34. Mattiazzi AD. Evaluación clínica del acceso vascular. Rev Nefrol Diál Traspl. 1999;(48):17-25.

35. Beathard GA. An algorithm for the physical examination of early fistula failure. Semin Dial. 2005;18(4):331-5.

36. Kian K, Vassalotti JA. The new arteriovenous fistula: the need for earlier evaluation and intervention. Semin Dial. 2005;18(1):3-7.

37. Asif A, Roy-Chaudhury P, Beathard GA. Early arteriovenous fistula failure: a logical proposal for when and how to intervene. Clin J Am Soc Nephrol. 2006;1(2):332-9.

38. Ohira S, Naito H, Amano I, Azuma N, Ikeda K, Kukita K, et al.; Japanese Society for Dialysis Therapy. 2005 Japanese Society for Dialysis Therapy guidelines for vascular access construction and repair for chronic hemodialysis. Ther Apher Dial. 2006;10(5):449-62.

39. Jiménez Almonacid $P$, Enrique Gruss $E$, Lorenzo $S$, Lasala $M$, Hernández T, Portolés J, et al. Definición de procesos e indicadores para la gestión de accesos vasculares para hemodiálisis. Cir Esp Ed Impr. 2007;81(5):257-63.

40. Levine Ml. A challenge for nephrologists-increasing fistula maturation rates, reducing fistula maturation time, and decreasing dialysis catheter prevalence in the United States. Semin Dial. 2008;21(3):280-4.

41. McCann M, Einarsdóttir H, Van Waeleghem JP, Murphy F, Sedgewick J. Vascular access management 1: an overview. J Ren Care. 2008;34(2):77-84

42. Nassar GM. Endovascular management of the "failing to mature" arteriovenous fistula. Tech Vasc Interv Radiol. 2008;11(3):175-80.

43. Malovrh M. Non-matured arteriovenous fistulae for haemodialysis: diagnosis, endovascular and surgical treatment. Bosn J Basic Med Sci. 2010;10 Suppl 1:S13-7.

44. Neves Junior MA, Melo RC, Almeida CC de, Fernandes AR, Petnys A, Iwasaki ML, et al. Avaliação da perviedade precoce das fístulas arteriovenosas para hemodiálise. J Vasc Bras. 2011;10(2):105-9.

45. Ahmed GM, Mansour MO, Elfatih M, Khalid KE, Ahmed Ml. Outcomes of arteriovenous fistula for hemodialysis in Sudanese patients: singlecenter experience. Saudi J Kidney Dis Transpl. 2012;23(1):152-7.

46. Rose DA, Sonaike E, Hughes K. Hemodialysis access. Surg Clin North Am. 2013;93(4):997-1012.
47. Salimi F, Majd Nassiri G, Moradi M, Keshavarzian A, Farajzadegan Z, Saleki M, et al. Assessment of effects of upper extremity exercise with arm tourniquet on maturity of arteriovenous fistula in hemodialysis patients. J Vasc Access. 2013;14(3):239-44.

48. Sousa CN, Apóstolo JL, Figueiredo MH, Martins MM, Dias VF. Physical examination: how to examine the arm with arteriovenous fistula. Hemodial Int. 2013;17(2):300-6.

49. Huang SG, Rowe VL, Weaver FA, Hwang F, Woo K. Compliance with surgical follow-up does not influence fistula maturation in a county hospital population. Ann Vasc Surg. 2014;28(8):1847-52.

50. Malovrh M. Postoperative assessment of vascular access. J Vasc Access. 2014;15 Suppl 7:S10-4.

51. López Alonso MT, Lozano Moledo V, Yuguero Ortiz A, Fontseré Baldellou N. Influencia del ejercicio físico en el desarrollo de fístulas arteriovenosas nativas. Enferm Nefrol. 2015;18(3):168-73.

52. Cheng Q, Zhao YJ. The reasons for the failure of the primary arteriovenous fistula surgery in patients with end-stage renal disease. J Vasc Access. 2015;16 Suppl 10:S74-7.

53. Mufty H, Claes K, Heye S, Fourneau I. Proactive surveillance approach to guarantee a functional arteriovenous fistula at first dialysis is worth. J Vasc Access. 2015;16(3):183-8.

54. Rosenberg JE, Yevzlin AS, Chan MR, Valliant AM, Astor BC. Prediction of Arteriovenous Fistula Dysfunction: can it be Taught? Semin Dial. 2015;28(5):544-7.

55. Pereira OR, Fernandes J S, Menegaz TN. Evalution of the radiocephalic fistula maturation for hemodialysis access. ACM Arq Catarin Med. 2016;45(2):2-10.

56. Martinez L, Esteve V, Yeste M, Artigas V, Llagostera S. Neuromuscular electrostimulation: a new therapeutic option to improve radio-cephalic arteriovenous fistula maturation in end-stage chronic kidney disease patients. Int Urol Nephrol. 2017;49(9):1645-52.

57. Rodrigues AT, Colugnati FA, Bastos MG. Evaluation of variables associated with the patency of arteriovenous fistulas for hemodialysis created by a nephrologist. J Bras Nefrol. 2018;40(4):326-32.

58. Siddiqui MA, Ashraff S, Carline T. Maturation of arteriovenous fistula: analysis of key factors. Kidney Res Clin Pract. 2017;36(4):318-28.

59. Vladimir F, Ahmad S, Shalhub S. Detecting pending hemodialysis access failure: the physical exam. In: Shalhub S, Dua A, Shin S, editors. Hemodialysis access. Cham: Springer; 2017. p. 183-90.

60. Robbin ML, Greene T, Cheung AK, Allon M, Berceli SA, Kaufman JS, et al.; Hemodialysis Fistula Maturation Study Group. Arteriovenous fistula development in the first 6 weeks after creation. Radiology. 2016;279(2):620-9

61. Flu H, Breslau PJ, Krol-van Straaten JM, Hamming JF, Lardenoye JW. The effect of implementation of an optimized care protocol on the outcome of arteriovenous hemodialysis access surgery. J Vasc Surg. 2008;48(3):659-68.

62. Blessios GA, Park JM, Barone KM. Effect of a rapid clinical protocol to the conversion from central venous hemodialysis catheter to arteriovenous access. J Vasc Access. 2016;17(2):124-30.

63. Dwyer A, Shelton P, Brier M, Aronoff G. A vascular access coordinator improves the prevalent fistula rate. Semin Dial. 2012;25(2):239_ 43.

64. Frazão CM, Fernandes MI, Nunes MG, Sá JD de, Lopes MV, Lira $\mathrm{AL}$. Components of a roy's adaptation model in patients undergoing hemodialysis. Rev Gaúcha Enferm. 2013;34(4):45-52. 